\title{
Tratamento medicamentoso e não medicamentoso em pacientes com transtorno do espectro autista: percepção de cuidadores
}

\author{
Drug and non-drug treatment in patients with autistic spectrum disorder: perception of caregivers \\ Tratamiento farmacológico y no farmacológico en pacientes con trastorno del espectro autista:
} percepción de los cuidadores

Recebido: 29/07/2021 | Revisado: 04/08/2021 | Aceito: 06/08/2021 | Publicado: 10/08/2021

Isabel Fiuza Menezes da Silva

ORCID: https://orcid.org/0000-0001-5588-5051

Centro Universitário de Patos, Brasil isabelsilva@med.fiponline.edu.br

Milena Nunes Alves de Sousa

ORCID: https://orcid.org/0000-0002-9431-6326

Centro Universitário de Patos, Brasil milenanunes@ fiponline.edu.br

\begin{abstract}
Resumo
Introdução: O transtorno do espectro autista (TEA) se caracteriza por déficits clinicamente relevantes e permanentes na comunicação social e interações sociais. Limitações de comunicação verbal e não verbal, falta de reciprocidade social, relacionamentos de amizades comprometidos para o estágio de desenvolvimento, comportamentos restritos e repetitivos, sendo esses comportamentos motores e verbais estereotipados ou os sensoriais incomuns, exagerada aderência a rotinas e padrões ritualizados, interesses resumidos e intensos. Objetivo: Analisar os efeitos do tratamento medicamentoso e não medicamentoso dos pacientes com transtorno do espectro autista (TEA) na visão nos cuidadores. Métodos: Pesquisa de campo, descritiva e quantitativa, realizada com 59 cuidadores das regiões do Sertão da Paraíba e do Cariri no Ceará. A coleta de dados foi efetivada com um questionário online elaborado no Google Forms, em que os cuidadores das crianças com TEA receberam o link por meio de grupos de mensagens instantâneas (WhatsApp) das regiões citadas. Para a análise dos dados foram utilizados testes descritivos de medidas de tendência central e de dispersão, bem como o teste de Regressão Logística Binária (método enter). Resultados: A idade média das crianças era de 6,25 anos, a maioria do sexo masculino $(94,9 \%)$, com diagnóstico de TEA fechado $(89,8 \%)$ e começaram a frequentar a escola entre $0-3$ anos de idade $(76,3 \%)$. Entre as medicações, a que se mostrou mais utilizada foi a risperidona $(40,7 \%)$. A maioria relatou que as medicações apresentam efeitos colaterais $(62,5 \%)$, entre estes, o aumento do apetite $(28,8 \%)$. Além disso, $88,1 \%$ dos cuidadores relataram que os pacientes utilizavam tratamento não medicamentoso, considerando-o como aquele que proporciona melhor qualidade de vida $(76,3 \%)$. Houve associações estatisticamente significativas entre tratamento medicamentoso, comportamento agressivo $\left(\chi^{2}(1)=\right.$ 6,133, $p<0,013, \phi=-0,322)$, hiperatividade $\left(\chi^{2}(1)=8,854, p<0,003, \phi=-0,387\right)$ e qualidade do sono $\left(\chi^{2}(1)=27,717\right.$, $p<0,0001, \phi=-0,345)$. Além disso, o tratamento não medicamentoso foi associado à terapia ocupacional-integral sensorial $\left(\chi^{2}(1)=3,622, p<0,057, \phi=-0,248\right)$. Conclusão: $\mathrm{O}$ tratamento não medicamentoso foi apontado como o de maior resposta, propiciando melhorias dos sintomas nucleares, com melhora da qualidade de vida, além de que não trazer efeitos colaterais, enquanto que o medicamentoso está relacionado com o tratamento de sintomas específicos, podendo trazer efeitos colaterais.
\end{abstract}

Palavras-chave: Transtorno do espectro autista; Autismo; Tratamentos; Terapia não medicamentosa.

\begin{abstract}
Introduction: Autism Spectrum Disorder (ASD) is characterized by clinically relevant and permanent deficits in social communication and social interactions. Limitations of verbal and non-verbal communication, lack of social reciprocity, friendship relationships compromised for the developmental stage, restricted and repetitive behaviors, being these stereotyped motor and verbal behaviors or unusual sensory ones, exaggerated adherence to ritualized routines and patterns, summarized interests and intense. Objective: To analyze the effects of drug and non-drug treatment of patients with autism spectrum disorder (ASD) on the vision of caregivers. Methods: Field research,
\end{abstract}


descriptive and quantitative, carried out with 59 caregivers from the Sertão da Paraíba and Cariri regions in Ceará. Data collection was carried out with an online questionnaire prepared on Google Forms, in which caregivers of children with ASD received the link through instant messaging groups (WhatsApp) in the regions mentioned. For data analysis, descriptive tests of measures of central tendency and dispersion were used, as well as the Binary Logistic Regression test (enter method). Results: The average age of the children was 6.25 years, most were male (94.9\%), diagnosed with closed ASD (89.8\%) and started attending school between 0-3 years old. (76.3\%). Among the medications, the one that was most used was rispiridone (40.7\%). Most reported that medications have side effects (62.5\%), including increased appetite (28.8\%). In addition, $88.1 \%$ of caregivers reported that patients used non-drug treatment, considering it as the one that provides better quality of life (76.3\%). There were statistically significant associations between drug treatment, aggressive behavior $\left(\chi^{2}(1)=6.133, \mathrm{p}<0.013, \phi=-0.322\right)$, hyperactivity $\left(\chi^{2}(1)\right.$ $=8.854, \mathrm{p}<0.003, \phi=-0.387)$ and quality of sleep $\left(\chi^{2}(1)=27,717, \mathrm{p}<0.0001, \phi=-0.345\right)$. In addition, non-drug treatment was associated with sensory-integral occupational therapy $\left(\chi^{2}(1)=3.622, p<0.057, \phi=-0.248\right)$. Conclusion: Non-drug treatment was appointed as the most responsive, providing improvements in core symptoms, with improved quality of life, in addition to not causing side effects, while drug therapy is related to the treatment of specific symptoms, and may bring Side effects.

Keywords: Autism spectrum disorder; Autism; Treatments; Non-drug therapy.

\section{Resumen}

Introducción: El trastorno del espectro autista (TEA) se caracteriza por déficits permanentes y clínicamente relevantes en la comunicación social y las interacciones sociales. Limitaciones de la comunicación verbal y no verbal, falta de reciprocidad social, relaciones de amistad comprometidas para la etapa de desarrollo, conductas restringidas y repetitivas, ya sean conductas motoras y verbales estereotipadas o sensoriales inusuales, adherencia exagerada a rutinas y patrones ritualizados, intereses resumidos y intenso. Objetivo: Analizar los efectos del tratamiento farmacológico y no farmacológico de pacientes con trastorno del espectro autista (TEA) sobre la visión de los cuidadores. Métodos: Investigación de campo, descriptiva y cuantitativa, realizada con 59 cuidadores de las regiones Sertão da Paraíba y Cariri en Ceará. La recolección de datos se realizó con un cuestionario en línea elaborado en Google Forms, en el cual los cuidadores de niños con TEA recibieron el enlace a través de grupos de mensajería instantánea (WhatsApp) en las regiones mencionadas. Para el análisis de los datos se utilizaron pruebas descriptivas de medidas de tendencia central y dispersión, así como la prueba de Regresión Logística Binaria (método enter). Resultados: La edad promedio de los niños fue de 6.25 años, la mayoría eran varones (94.9\%), diagnosticados de TEA cerrado (89.8\%) y comenzaron a asistir a la escuela entre los 0-3 años (76.3\%). Entre los medicamentos, el más utilizado fue la rispiridona $(40,7 \%)$. La mayoría informó que los medicamentos tienen efectos secundarios $(62,5 \%)$, incluido un aumento del apetito (28,8\%). Además, el $88,1 \%$ de los cuidadores refirió que los pacientes utilizaban un tratamiento no farmacológico, considerándolo como el que proporciona una mejor calidad de vida $(76,3 \%)$. Fue observado asociaciones estadísticamente significativas entre tratamiento farmacológico, comportamiento agresivo $\left(\chi^{2}\right.$ $(1)=6.133, \mathrm{p}<0.013, \phi=-0.322)$, hiperactividad $\left(\chi^{2}(1)=8.854, \mathrm{p}<0.003, \phi=-0.387\right)$ y calidad del sueño $\left(\chi^{2}(1)=\right.$ $27,717, \mathrm{p}<0,0001, \phi=-0,345)$. Además, el tratamiento no farmacológico se asoció con la terapia ocupacional integral sensorial $\left(\chi^{2}(1)=3.622, \mathrm{p}<0.057, \phi=-0.248\right)$. Conclusão: $\mathrm{O}$ tratamento não medicamentoso foi apontado como o de maior resposta, propiciando melhorias dos sintomas nucleares, com melhora da qualidade de vida, além de que não trazer efeitos colaterais, enquanto que o medicamentoso está relacionado com o tratamento de sintomas específicos, podendo trazer efectos colaterales.

Palabras clave: Trastorno del espectro autista; Autismo; Tratos; Terapia no farmacológica.

\section{Introdução}

O Transtorno do Espectro Autista (TEA) se caracteriza por déficits clinicamente relevantes e permanentes na comunicação social e interações sociais. Limitações de comunicação verbal e não verbal, falta de reciprocidade social, relacionamentos de amizades comprometidos para o estágio de desenvolvimento, comportamentos restritos e repetitivos, sendo esses comportamentos motores e verbais estereotipados ou os sensoriais incomuns, exagerada aderência a rotinas e padrões ritualizados, interesses resumidos e intensos (Nikolov, Jonker \& Scahill, 2006; American Psychiatric Association - APA, 2013; Masi, Demayo, Glozier \& Guastella, 2017; Genovese \& Butler, 2018). 
Ademais, o TEA é um transtorno neurológico, presente desde o nascimento ou começo da infância, porém pode não se manifestar devido ao intenso apoio e cuidados dos pais ou cuidadores nos primeiros anos de vida ou até que a sociedade os imponha. Além disso, introduziu-se à categoria do TEA, o transtorno de Asperger, transtorno desintegrativo da infância e transtorno global ou invasivo do desenvolvimento sem outra especificação (Nikolov, Jonker \& Scahill, 2006; APA, 2013; Masi, Demayo, Glozier \& Guastella, 2017).

O TEA faz parte de um grupo de condições definidas como transtornos invasivos do desenvolvimento (TIDs). Uma característica comum dos TIDs é um comprometimento global nas várias áreas do funcionamento: a interação social, a comunicação e a presença de comportamentos repetitivos e interesses restritos. Esses déficits se desenvolvem com relação à idade mental do indivíduo e, geralmente, tornam-se evidentes no terceiro ano de vida e frequentemente pode vir com algum grau de deficiência intelectual (Nikolov, Jonker \& Scahill, 2006; Masi, Demayo, Glozier \& Guastella, 2017; Genovese \& Butler, 2018).

As comorbidades genéticas e ambientais são detectadas em pelo menos $20 \%$ dos indivíduos com Transtornos do Espectro do Autismo (TEA). Os fatores associados aos quadros de TEA estão nessa categoria: exposição pré-natal a teratógenos; complicações perinatais como prematuridade, hemorragia intracraniana; anoxia; infecções; e síndromes genéticas cromossômicas e gênicas. Essas comorbidades representam a maior parte síndromes genéticas. Dessa forma, o possível envolvimento de fatores genéticos na etiologia do TEA é reconhecido há muito tempo (Muhle, Trentacoste \& Rapin, 2004; Owen-Deschryver, Carr, Cale e Blakeley-Smith (2008).

Baseado nas evidências revisadas, a prevalência do autismo é de 62/10.000 de acordo com a mediana das estimativas (Elsabbagh et al., 2012) com uma incidência quatro vezes maior em meninos do que em meninas (Fombonne, 2009). Já se tratando da proporção de incidência entre indivíduos do sexo masculino e feminino, estudos indicam que o autismo é quatro vezes mais prevalente em meninos do que em meninas em indivíduos com níveis normais de inteligência (Newsom \& Hovanitz, 2006; Owen-Deschryver, Carr, Cale e Blakeley-Smith (2008).

A variabilidade em meio à gravidade no comportamento, cognição e mecanismos biológicos do autismo é muito heterogênea, sendo assim aproximadamente $60-70 \%$ possuem algum grau de deficiência intelectual, enquanto os possuidores de autismo leve apresentam faixa normal de inteligência e cerca de $10 \%$ dos portadores de TEA têm excelentes habilidades intelectuais condizentes para a idade (Brentani et al., 2013; Masi, Demayo, Glozier \& Guastella, 2017).

Este contexto demandou a observação de fontes múltiplas para as informações sobre as manifestações de sintomas para os comportamentos repetitivos e interesses fixos e focados, com a observação clínica especializada e os relatos dos pais, cuidadores e professores de significativa relevância. Dessa maneira, considerou-se um diagnóstico mais estável do Transtorno do Espectro Autista (TEA) e uma possível diferenciação mais segura dentre outros transtornos, quando as fontes múltiplas adicionaram os pais, cuidadores e professores observando os pacientes em uma rotina diária e prolongada para assim identificar uma história de interesses fixos, rotinas ou rituais e comportamento repetitivos (APA, 2013; Masi, Demayo, Glozier \& Guastella, 2017; Genovese \& Butler, 2018).

Nos últimos anos, estudos genéticos e fatores modulados ambientalmente destacaram a natureza poligênica dessas condições, mas a etiologia continua indefinida na maioria dos casos de TEA. A conectividade reduzida em várias áreas do cérebro é explicada por estudos de neuroimagem de anatomia e conectividade funcional que demonstram que essa patologia cerebral não se encontra localizada (Benvenuto, Battan, Benassi, Gialloreti \& Curatolo, 2016).

A complexidade do TEA é também um reflexo da falta de tratamentos eficazes para reverter por completo o núcleo dos sintomas dos distúrbios, bem como um estabelecimento de uma padronização de atendimento para todos os portadores. Entre as intervenções aplicadas, principalmente as baseadas em comportamentos e princípios de desenvolvimento, têm demonstrado eficácia na melhora dos sintomas globais dos autistas e um avanço na parte de linguagem cognitiva e habilidades 
adaptativas. Ademais, intervenções intensivas e precoces são capazes de trazer resultados mais positivos para os portadores de TEA. Porém devido à grande heterogeneidade do transtorno e a sua natureza torna se improvável um tratamento específico, como também inadequado uma única abordagem terapêutica para todos os portadores, o que demanda planos terapêuticos individualizados e multidisciplinares (Benvenuto, Battan, Benassi, Gialloreti \& Curatolo, 2016; Genovese \& Butler, 2018; Postorino et al., 2018; Magalhães et al., 2021).

A terapêutica deve ser elaborada de acordo com a faixa etária do paciente. Assim, com crianças pequenas, a prioridade deve ser a terapia da fala, da interação social/linguagem, educação especial e suporte familiar; com os adolescentes, os alvos seriam os grupos de habilidades sociais, terapia ocupacional e sexualidade. O sucesso terapêutico é dependente da experiência e conhecimento dos profissionais sobre a temática do autismo e a habilidade de trabalhar em equipe com a família e cuidadores (Bosa, 2006).

As crianças com TEA precisam de tratamentos individualizados e intensivos e para isso são necessários centros especializados em autismo que abordem intervenções comportamentais, psicológicas e educacionais (Babatin, Alzahrani, Jan, Alkarimi \& Jan, 2016).

Normalmente, os tratamentos de primeira linha para as crianças com TEA são os psicossociais e intervenções educacionais com o intuito de estimular a aquisição da linguagem, melhorar habilidades sociais e de comunicação e minimizar os comportamentos mal adaptativos. Já quando se introduz intervenções farmacológicas na terapêutica individualizada de cada paciente busca-se o alvo de sintomas específicos que acompanham os sintomas nucleares, pois inexistem medicações disponíveis para o tratamento (Nikolov, Jonker \& Scahill, 2006; Genovese \& Butler, 2018; Hyman, Levy \& Myers, 2019).

Os agentes farmacológicos usados para sintomas específicos (comportamentos autodestrutivos, rituais compulsivos, baixa tolerância a frustações com excessos explosivos, hiperatividade e outros) são pertencentes a vários grupos de medicações. Porém, ainda que a medicação melhore a vida de alguns pacientes, seus benefícios são restritos e há dúvidas quanto à resposta positiva dos pacientes. Outro fator da medicação é que em longo prazo os benefícios ainda são desconhecidos e parte dos pacientes descontinua a medicação por perda da eficácia ou devido os efeitos colaterais (Nikolov, Jonker \& Scahill, 2006; Masi, Demayo, Glozier \& Guastella, 2017; Genovese \& Butler, 2018; Hyman, Levy \& Myers, 2019).

Existem alguns tratamentos medicamentosos para o Transtorno do Espectro Autista (TEA), esses são compostos por grandes categorias de fármacos. Porém nenhum se mostrou eficaz para as características essenciais do TEA. É feito o uso de medicamentos psicotrópicos para direcionar os sintomas psiquiátricos em crianças, adolescentes e adultos com problemas de desenvolvimento (Cukier \& Barrios, 2019).

As grandes categorias de fármacos comumente utilizadas são os antipsicóticos atípicos (clozapina, a risperidona, a olanzapina, a quetiapina, a ziprazidona e o aripiprazol), antipsicóticos típicos (haloperidol e a clorpromazina), os inibidores da recaptação da serotonina (clomipramina, fluoxetina, fluvoxamina, sertralina, paroxetina, citalopram e escitalopram) e estabilizadores do humor (carbonato de lítio, divalproato sódico) (Nikolov, Jonker \& Scahill, 2006; Hyman, Levy \& Myers, 2019).

Conforme Schwartzman e Araújo (2014), apesar de inexistir cura para TEA, os pacientes expostos a tratamento precoce podem ter prejuízos amenizados. Ainda afirma que para que se consiga um tratamento precoce é necessário um diagnóstico bem na fase inicial da vida que é feito por uma equipe multifatorial (neuropediatra, psicólogos, psiquiatras, psicopedagogos, fonoaudiólogos), para que se inicie uma intervenção educacional especializada. Dessa maneira, poderá ser elaborado um plano de intervenção com evidência científica de eficácia e aplicabilidade, de acordo com as necessidades individuais de cada paciente com TEA (Schwartzman \& Araújo, 2014).

O quadro sinótico apresentado indica a relevância do tema para atualidade. Ganhando espaço científico maior nos últimos tempos, torna-se necessário maior engajamento por parte dos profissionais para se mostrarem preparados ao se deparar 
com pacientes com TEA e conseguirem traçar desde um diagnóstico precoce até montar um plano individualizado de terapêutica para seus pacientes. Já para os cuidadores é de suma importância estudos sobre a temática para mostrá-los uma jornada de exemplos de crescimento no desenvolvimento e um norte a ser seguido que traga bons resultados às crianças com TEA, lembrando sempre que os cuidadores fazem parte do tratamento e mostram-se como ferramenta indispensável nas estimulações diárias dos autistas e esses precisam estar bem preparados.

Dessa forma, podem-se trazer melhores respostas ao desenvolvimento dos pacientes com a soma dos profissionais aos cuidadores como equipe. Ainda, mostra-se importante um estudo/pesquisa quanto às repostas aos tratamentos não medicamentosos e medicamentosos já disponíveis para analisarmos as respostas dessas terapêuticas e dessa forma, nortear os planos terapêuticos com evidências de bons resultados já alcançados.

\section{Metodologia}

O presente estudo trata-se de uma pesquisa de campo descritiva com caráter quantitativo, realizada no Sertão da Paraíba e na região do Cariri no Ceará. A pesquisa quantitativa centra-se na objetividade, na coleta e análise dos dados obtidos, permitindo quantificar os resultados observados, o que possibilita um enfoque maior na interpretação do alvo de pesquisa dando importância ao contexto no qual os dados foram colhidos. Utiliza-se de procedimentos estruturados e instrumentos formais para a coleta dos dados e analisa-os mediante procedimentos estatísticos (Gerhardt \& Silveira, 2009).

A coleta de dados foi efetivada entre os meses de fevereiro a junho de 2021, a partir da aplicação de um questionário online elaborado pelas pesquisadoras e disponibilizado pelo Google Forms, em que os cuidadores das crianças com transtorno do espectro autista (TEA) receberam o link por meio de dois grupos de autismo das regiões envolvidas na pesquisa por um aplicativo de mensagens instantâneas (WhatsApp). A pesquisa englobou cuidadores das cidades Juazeiro do Norte, Crato, Várzea Alegre, Missão Velha, Barbalha e Mauriti, ambas localizadas na região do Cariri cearense, que formaram o Grupo Autismo e cuidadores de cidades da Paraíba, de Pernambuco e da Bahia, que formaram o grupo Autismo no Sertão, compreendendo as cidades de Tabira, Afogados da Ingazeira, São José do Egito, Santa Terezinha, Campina Grande, Nova Olinda, Patos, Teixeira de Freitas e Carnaíba. O universo de pesquisa contemplava, aproximadamente, 150 cuidadores.

Para a seleção da amostra adotaram-se os seguintes critérios: Ser cuidador de crianças com autismo e participar dos grupos de autismo do Sertão da Paraíba e da Região do Cariri, onde serão aplicados o instrumento da pesquisa. Como critério de exclusão tem-se o preenchimento incompleto ou incorreto do instrumento de pesquisa. Participaram, assim, 59 cuidadores (40\% da população de pesquisa).

Os dados foram analisados por meio do software Statistical Package for the Social Sciences (SPSS), versão 25. Foram utilizados testes descritivos de medidas de tendência central (média e mediana) e medidas de dispersão (desvio padrão). Utilizou-se o teste de Regressão Logística Binária (método enter) para a predição de valores do modelo e o qui-quadrado de independência $(2 \times 2$ e 2x4) para verificar a associação e comparação entre grupos. A significância estatística foi de $\mathrm{p} \leq 0,05$.

\section{Resultados}

Conforme a amostra de cuidadores, as crianças com Transtorno do Espectro Autista (TEA), tinham média de idade de $6,25(\mathrm{DP}=2,08)$ anos. Ainda, em conformidade com a Tabela 1, a maioria dos pacientes era do sexo masculino $(94,9 \%)$, tinha diagnóstico de TEA fechado (89,8\%) e começou a frequentar a escola entre 0-3 anos de idade (76,3\%). Quanto à família, a maioria tinha um filho com autismo $(91,5 \%)$ e renda mensal de até 1 salário mínimo $(35,6 \%)$. 
Tabela 1. Descrição demográfica dos dados categóricos.

\begin{tabular}{|c|c|c|}
\hline Variáveis & Frequência Absoluta $(F)$ & Frequência relativa $(\%)$ \\
\hline \multicolumn{3}{|l|}{ Sexo } \\
\hline Masculino & 56 & 94,9 \\
\hline Feminino & 3 & 5,1 \\
\hline \multicolumn{3}{|l|}{ A criança possui diagnóstico fechado } \\
\hline Sim & 53 & 89,8 \\
\hline Não & 6 & 10,2 \\
\hline \multicolumn{3}{|l|}{ Quando começou a frequentar a escola } \\
\hline $0-3$ anos & 45 & 76,3 \\
\hline 3-4 anos & 12 & 20,3 \\
\hline 4-5 anos & 2 & 3,4 \\
\hline \multicolumn{3}{|c|}{ Quantos filhos na família apresentam autismo } \\
\hline Um filho & 54 & 91,5 \\
\hline Dois filhos & 5 & 8,5 \\
\hline \multicolumn{3}{|l|}{ Renda familiar } \\
\hline O a 1 salário mínimo & 21 & 35,6 \\
\hline 1 a 2 salários mínimos & 15 & 25,4 \\
\hline 2 a 3 salários mínimos & 6 & 10,2 \\
\hline 3 a 4 salários mínimos & 3 & 5,1 \\
\hline 4 a 5 salários mínimos & 6 & 10,2 \\
\hline Mais de 5 salários mínimos & 8 & 13,6 \\
\hline
\end{tabular}

Fonte: Dados de Pesquisa (2021).

Na Tabela 2 são apresentados dados referentes à percepção dos cuidadores acerca do tratamento medicamentoso e não medicamentoso. Entre as medicações, a que se mostrou mais utilizada foi a risperidona (40,7\%). A maioria relatou que as medicações apresentam efeitos colaterais (62,5\%), entre estes, o aumento do apetite (28,8\%). Além disso, 88,1\% dos participantes relataram que os pacientes utilizavam tratamento não medicamentoso, à medida que consideraram este o tratamento que proporciona melhor qualidade de vida $(76,3 \%)$. 
Tabela 2. Descrição categórica do tratamento medicamentoso e não medicamentoso.

\begin{tabular}{|c|c|c|}
\hline Variáveis & Frequência Absoluta (F) & Frequência relativa $(\%)$ \\
\hline \multicolumn{3}{|l|}{ Faz uso de medicação } \\
\hline Sim & 34 & 57,6 \\
\hline Não & 25 & 42,4 \\
\hline \multicolumn{3}{|l|}{ Risperidona } \\
\hline$N \tilde{a} o$ & 35 & 59,3 \\
\hline $\operatorname{Sim}$ & 24 & 40,7 \\
\hline \multicolumn{3}{|l|}{ Quetiapina } \\
\hline$N \tilde{a} o$ & 58 & 98,3 \\
\hline $\operatorname{Sim}$ & 1 & 1,7 \\
\hline \multicolumn{3}{|l|}{ Aripiprazol } \\
\hline Não & 55 & 93,2 \\
\hline $\operatorname{Sim}$ & 4 & 6,8 \\
\hline \multicolumn{3}{|l|}{ Fluoxetina } \\
\hline Não & 58 & 98,3 \\
\hline $\operatorname{Sim}$ & 1 & 1,7 \\
\hline \multicolumn{3}{|l|}{ Sertralina } \\
\hline$N \tilde{a} o$ & 58 & 98,3 \\
\hline $\operatorname{Sim}$ & 1 & 1,7 \\
\hline \multicolumn{3}{|l|}{ Melatonina } \\
\hline$N \tilde{a} o$ & 53 & 89,8 \\
\hline $\operatorname{Sim}$ & 6 & 10,2 \\
\hline \multicolumn{3}{|l|}{ Efeitos colaterais } \\
\hline Sim & 25 & 62,5 \\
\hline$N \tilde{a} o$ & 15 & 37,5 \\
\hline \multicolumn{3}{|l|}{ Ganho de peso } \\
\hline Não & 43 & 72,9 \\
\hline Sim & 16 & 27,1 \\
\hline \multicolumn{3}{|l|}{ Tontura } \\
\hline$N \tilde{a} o$ & 56 & 94,9 \\
\hline $\operatorname{Sim}$ & 3 & 5,1 \\
\hline \multicolumn{3}{|l|}{ Insônia } \\
\hline Não & 55 & 93,2 \\
\hline Sim & 4 & 6,8 \\
\hline \multicolumn{3}{|l|}{ Maior apetite } \\
\hline Não & 42 & 71,2 \\
\hline $\operatorname{Sim}$ & 17 & 28,8 \\
\hline \multicolumn{3}{|l|}{ Redução de apetite } \\
\hline Não & 54 & 91,5 \\
\hline Sim & 5 & 8,5 \\
\hline \multicolumn{3}{|l|}{ Sedação transitória } \\
\hline Não & 55 & 93,2 \\
\hline $\operatorname{Sim}$ & 4 & 6,8 \\
\hline \multicolumn{3}{|c|}{ Tratamento não medicamentoso } \\
\hline Sim & 52 & 88,1 \\
\hline Não & 7 & 11,9 \\
\hline \multicolumn{3}{|l|}{ Qual melhor tratamento } \\
\hline Medicamentoso & 14 & 23,7 \\
\hline Não medicamentoso & 45 & 76,3 \\
\hline
\end{tabular}


O modelo de regressão logística binária (método enter) entre o uso de medicação (sim ou não), benefícios no tratamento medicamentoso ( $\operatorname{sim}$ ou não) e efeitos colaterais ( $\operatorname{sim}$ ou não) foi estatisticamente significativo $\left[\mathrm{c}^{2}(2)=6,954, p<\right.$ 0,03]. Este previu adequadamente 88,9\% dos casos (sendo 100,0\% dos casos corretamente classificados para os pacientes que fazem uso de medicação e $0,0 \%$ dos casos corretamente classificados para os pacientes que não fazem uso de medicação) (Tabela 3).

Também se verificaram associações entre o tratamento não medicamentoso (sim ou não), malefícios no tratamento não medicamentoso ( $\operatorname{sim}$ ou não) e fazer uso de medicação ( $\operatorname{sim}$ ou não). O modelo foi estatisticamente significativo [ ${ }^{2}(3)=$ 9,720, $p<0,02$ ], prevendo adequadamente 91,5\% dos casos (sendo 98,1\% dos casos corretamente classificados para os pacientes que fazem tratamento não medicamentoso e 42,9\% dos casos corretamente classificados para os pacientes que não fazem tratamento não medicamentoso) (Tabela 3).

Tabela 3. Classificações previstas pelo modelo.

\begin{tabular}{|c|c|c|c|c|}
\hline & & \multicolumn{3}{|c|}{ Valores Preditos } \\
\hline & & \multicolumn{2}{|c|}{ Faz uso de medicação } & \multirow[b]{2}{*}{ Classificações corretas (\%) } \\
\hline \multicolumn{2}{|l|}{ Valores Observados } & Não & Sim & \\
\hline \multirow[t]{2}{*}{ Faz uso de medicação } & Não & 0 & 4 & 0,0 \\
\hline & Sim & 0 & 32 & 100,0 \\
\hline \multicolumn{2}{|l|}{ Classificação correta (total) } & & & 88,9 \\
\hline & & \multicolumn{3}{|c|}{ Valores Preditos } \\
\hline & & \multicolumn{2}{|c|}{$\begin{array}{c}\text { Faz tratamento não } \\
\text { medicamentoso }\end{array}$} & \\
\hline \multicolumn{2}{|l|}{ Valores Observados } & Não & Sim & Classificações corretas (\%) \\
\hline \multirow[t]{2}{*}{ Faz tratamento não medicamentoso } & Não & 3 & 4 & 42,9 \\
\hline & Sim & 1 & 51 & 98,1 \\
\hline Classificação correta (total) & & & & 91,5 \\
\hline
\end{tabular}

Fonte: Dados de Pesquisa (2021).

Tendo em vista os preditores analisados, apenas benefícios no tratamento medicamentoso teve impacto estatisticamente significativo $(\exp (b)=-3,021$ [95\% IC: 0,004-0,594), revelando que menos de um ponto no escore de benefícios no tratamento medicamentoso ( $\operatorname{sim}$ ) aumenta em 0,04 vezes as chances de o paciente fazer uso de medicação. Quanto à malefícios no tratamento não medicamentoso, este teve impacto estatisticamente significativo $(\exp (b)=0,048[95 \%$ IC: $0,006-0,353$ ), demonstrando que menos de um ponto no escore de malefícios no tratamento não medicamentoso (não) aumenta em 0,04 vezes as chances de o paciente fazer uso do tratamento (Tabela 4). 
Tabela 4. Variáveis preditoras do uso de medicação e tratamento não medicamentoso.

\begin{tabular}{|c|c|c|c|c|c|c|}
\hline \multirow[b]{2}{*}{ Uso de medicação } & \multirow[b]{2}{*}{ Wald } & \multirow[b]{2}{*}{ df } & \multirow[b]{2}{*}{ Sig. } & \multirow[b]{2}{*}{$\operatorname{Exp}(B)$} & \multicolumn{2}{|c|}{$95 \%$ C.I.for $\operatorname{EXP}(B)$} \\
\hline & & & & & $\begin{array}{l}\text { Limite } \\
\text { Inferior }\end{array}$ & Limite Superior \\
\hline Benefícios no tratamento & 5,611 & 1 & 0,018 & 0,049 & 0,004 & 0,594 \\
\hline Efeitos colaterais & 0,096 & 1 & 0,757 & 1,528 & 0,105 & 22,347 \\
\hline Constant & 0,222 & 1 & 0,637 & 0,552 & - & - \\
\hline & & & & & \multicolumn{2}{|c|}{ 95\% C.I.for EXP(B) } \\
\hline $\begin{array}{l}\text { Tratamento não } \\
\text { medicamentoso }\end{array}$ & Wald & df & Sig. & $\operatorname{Exp}(B)$ & $\begin{array}{l}\text { Limite } \\
\text { Inferior }\end{array}$ & Limite Superior \\
\hline Malefícios no tratamento (não) & 8,893 & 1 & 0,003 & 0,048 & 0,006 & 0,353 \\
\hline Faz uso de medicação & 0,038 & 1 & 0,845 & 1,201 & 0,192 & 7,503 \\
\hline Constant & 0,012 & 1 & 0,914 & 0,912 & - & - \\
\hline
\end{tabular}

Nota: Wald = teste de Wald; $\mathrm{df}=$ graus de liberdade; Sig. = significância estatística; $\operatorname{Exp}(\mathrm{B})=$ razão de chance. Fonte: Dados de Pesquisa (2021).

Houve associações estatisticamente significativas entre tratamento medicamentoso, comportamento agressivo $\left(\chi^{2}(1)=\right.$ $6,133, p<0,013, \phi=-0,322)$, hiperatividade $\left(\chi^{2}(1)=8,854, p<0,003, \phi=-0,387\right)$ e qualidade do sono $\left(\chi^{2}(1)=27,717, p<\right.$ $0,0001, \phi=-0,345)$. Além disso, o tratamento não medicamentoso foi associado à terapia ocupacional-integral sensorial $\left(\chi^{2}(1)\right.$ $=3,622, p<0,057, \phi=-0,248)$ (Tabela 5).

Análises de razão de chance (odds-ratio) revelaram que na percepção dos cuidadores, há 1,00 vezes mais chance de o tratamento medicamentoso não promover melhorias no comportamento agressivo. Pacientes que não fazem uso de medicação apresentam tendência a não perceberem melhorias no comportamento hiperativo e qualidade do sono, em comparação àqueles que fazem. Na visão dos cuidadores, há 0,22 vezes mais chance de pacientes que aderem ao tratamento não medicamentoso se beneficiarem com a terapia ocupacional - integral sensorial.

Com relação às variáveis sociodemográficas, foram verificadas associações estatisticamente significativas entre nível de escolaridade da mãe e tratamento não medicamentoso $\left(\chi^{2}(5)=14,850, p<0,011, \phi=0,502\right)$ (Tabela 5). Evidenciou-se que as mães dos pacientes que possuem ensino superior completo possuem mais chance de acatar o tratamento não medicamentoso, em comparação às mães que possuem ensino fundamental completo. 
Tabela 5. Estatísticas do qui-quadrado de independência $(2 \times 2$ e $2 \times 4)$ referentes ao tratamento medicamento e não medicamentoso.

\begin{tabular}{|c|c|c|c|c|c|c|}
\hline \multirow{2}{*}{\multicolumn{3}{|c|}{ Tratamento medicamentoso }} & \multicolumn{2}{|c|}{ Comportamento agressivo } & \multirow{2}{*}{\multicolumn{2}{|c|}{$\chi^{2}(g l)$}} \\
\hline & & & Não & Sim & & \\
\hline \multicolumn{3}{|c|}{ Sim } & 24 & 10 & \multirow{2}{*}{\multicolumn{2}{|c|}{$6,133(1)^{* *}$}} \\
\hline \multicolumn{3}{|c|}{ Não } & 24 & 1 & & \\
\hline \multirow{2}{*}{\multicolumn{3}{|c|}{ Tratamento medicamentoso }} & \multicolumn{2}{|c|}{ Comportamento hiperativo } & \multirow{2}{*}{\multicolumn{2}{|c|}{$\chi^{2}(g l)$}} \\
\hline & & & Não & Sim & & \\
\hline \multicolumn{2}{|c|}{ Sim } & & 24 & 10 & \multirow{2}{*}{\multicolumn{2}{|c|}{$8,854(1)^{* *}$}} \\
\hline \multicolumn{3}{|c|}{ Não } & 25 & 0 & & \\
\hline \multirow{2}{*}{\multicolumn{3}{|c|}{ Tratamento medicamentoso }} & \multicolumn{2}{|c|}{ Qualidade do sono } & \multirow{2}{*}{\multicolumn{2}{|c|}{$\chi^{2}(g l)$}} \\
\hline & & & Não & Sim & & \\
\hline \multicolumn{2}{|c|}{ Sim } & & 11 & 23 & \multirow{2}{*}{\multicolumn{2}{|c|}{$27,717(1)^{* *}$}} \\
\hline \multicolumn{3}{|c|}{ Não } & 25 & 0 & & \\
\hline & & & \multicolumn{2}{|c|}{ Terapia ocupacional } & & \\
\hline Tratamento nã & medicamentoso & & Não & Sim & & \\
\hline & & & 12 & 40 & 3,62 & $(1)^{* * *}$ \\
\hline & & & 4 & 3 & & \\
\hline & & Escolar & de da mãe & & & \\
\hline $\begin{array}{l}\text { Tratamento não } \\
\text { medicamentoso }\end{array}$ & $\begin{array}{c}\text { Fund. } \\
\text { incompleto }\end{array}$ & $\begin{array}{l}\text { Fund. } \\
\text { completo }\end{array}$ & $\begin{array}{c}\text { Médio } \\
\text { incompleto }\end{array}$ & $\begin{array}{c}\text { Médio } \\
\text { completo }\end{array}$ & $\begin{array}{c}\text { Superior } \\
\text { incompleto }\end{array}$ & $\begin{array}{l}\text { Superior } \\
\text { completo }\end{array}$ \\
\hline $\operatorname{Sim}(n)$ & 1 & 1 & 2 & 16 & 3 & 29 \\
\hline Resíduos ajustados & 0,4 & $-3,0^{* *}$ & 0,5 & $-1,4$ & $-0,8$ & $2,8 * *$ \\
\hline Não $(n)$ & 0 & 2 & 0 & 4 & 1 & 0 \\
\hline Resíduos ajustados & $-0,4$ & $3,0^{* *}$ & $-0,5$ & 1,4 & 0,8 & $-2,8 * *$ \\
\hline
\end{tabular}

Nota: ${ }^{* *} \mathrm{p}<0,01 ; \chi^{2}=$ qui-quadrado; $\mathrm{gl}=$ graus de liberdade; $n=$ número de casos. Fonte: Dados de Pesquisa (2021).

\section{Discussão}

Conforme os dados apresentados nessa pesquisa, a maioria dos pacientes do grupo amostral pertence ao sexo masculino, com diagnóstico de TEA fechado e começou a frequentar a escola entre 0-3 anos de idade. Quanto às famílias, a maioria tinha um filho com autismo e possuíam baixa renda mensal. Tais informações estão de acordo com dados identificados na literatura, como nos estudos de Mazi, DeMayo, Glozier e Guastella (2017), Baumer e Spence (2018), Consolini, Lopes e Lopes (2019), Hodges, Fealko e Soares (2020) e de Hyman, Levy e Myers, que evidenciaram uma maior prevalência no sexo masculino para o TEA e com início da vida escolar ainda nos primeiros anos de vida.

Consoante ao que foi observado neste estudo quanto à importância do diagnóstico precoce de distúrbios do autismo, para Schwartzman e Araújo (2014), apesar de inexistir cura para TEA, os pacientes expostos a tratamento precoce podem ter prejuízos amenizados. Ainda afirma que para que se consiga um tratamento precoce é necessário um diagnóstico na fase inicial da vida, o qual deve ser feito por uma equipe multifatorial (neuropediatra, psicólogos, psiquiatras, psicopedagogos, fonoaudiólogos), para que se inicie uma intervenção educacional especializada.

Portanto, poderá ser elaborado um plano de intervenção com evidência científica de eficácia e aplicabilidade, de acordo com as necessidades individuais de cada paciente com TEA (Genovese e Butler, 2018; Lord, Elsabbagh, Baird e Veenstra-Vanderweele, 2018). Ressalta-se que, uma vez estabelecido o diagnóstico precoce, as crianças com autismo devem ser avaliadas e gerenciadas para programas específicos para que haja rapidamente o início da terapêutica multidisciplinar, o que pode aumentar a probabilidade de uma boa resposta aos tratamentos ofertados (Babatin, Alzahrani, Jan, Alkarimi \& Jan, 2016; Lord, Elsabbagh, Baird \& Veenstra-Vanderweele, 2018). 
Intervir cedo também se refere em intervir na família e no sistema de cuidado e criação da criança, pois pesquisas acumuladas em programas de intervenção precoce mostraram que se as crianças não forem estimuladas no seu cotidiano, principalmente no que tange o mecanismo de estimulação central, nenhum benefício significativo seria obtido. Diante disso a Academia Americana de Pediatria recomenda uma vigilância do desenvolvimento em todas as consultas preventivas de saúde (Zalaquett, Schönstedt, Angeli, Herrrera \& Moyano, 2015; Lord, Elsabbagh, Baird \& Veenstra-Vanderweele, 2018).

Ao pensar que as interações sociais recíprocas facilitam a especialização cortical para informações sociais e linguísticas em um menor sem patologia de desenvolvimento subjacente, a falta de compromisso social e de comunicação associada com o TEA poderia ter efeitos colaterais mais negativos no próprio desenvolvimento do cérebro, dessa forma a intervenção precoce pode alterar a trajetória de desenvolvimento do cérebro em direção a um caminho de desenvolvimento mais adaptativo (Zalaquett, Schönstedt, Angeli, Herrrera \& Moyano, 2015; Genovese \& Butler, 2018; Lord, Elsabbagh, Baird \& Veenstra-Vanderweele, 2018).

Por conseguinte, ao se referir sobre a percepção dos cuidadores acerca do tratamento medicamentoso e nãomedicamentoso para o TEA, a categoria de fármacos que se mostrou mais predominante neste estudo foi a de antipsicóticos, seguido pelos inibidores seletivos da recaptação de serotonina (ISRS). Entretanto, de acordo com a amostra, não se mostraram eficazes para as características essenciais do TEA. Seu uso possui o intuito de direcionar os sintomas psiquiátricos em crianças com problemas de desenvolvimento. Tal informação é sustentada pelo estudo de Cukier e Barrios (2019).

Dados presentes na literatura reforçaram esse tratamento medicamentoso existente, consistindo em antipsicóticos atípicos (AAPs) que trazem a clozapina, a risperidona, a olanzapina, a quetiapina, a ziprazidona e o aripiprazol e os antipsicóticos tradicionais (típicos), como o haloperidol e a clorpromazina. Esses antipsicóticos possuem como alvo os sintomas de agressão, automutilação, destruição de propriedade, crise de ira e ataques de raiva, sintomas esses que foram apresentados por algumas crianças autistas que participaram desse estudo. Vale ressaltar que os AAPs têm menor risco de induzir efeitos colaterais neurológicos de curto prazo, como Parkinsonismo, e talvez discinesia tardia (DT) em longo prazo (Nikolov, Jonker \& Scahill, 2006; DeFilippis \& Wagner, 2016; Masi, Demayo, Glozier \& Guastella, 2017).

Entre as medicações utilizadas para o tratamento farmacológico das crianças com TEA nesse estudo, a que se mostrou mais utilizada foi a de antipsicóticos, em que a risperidona foi a mais citada, seguida por aripiprazol e quetiapina. Segundo dados da pesquisa de Nikolov, Jonker e Scahill (2006) a risperidona é muito usada, porém ainda que tenha sido documentada uma melhora significativa na hiperatividade e nas estereotipias, houve pouca evidência de benefícios nas características nucleares do autismo no curto prazo e estudos em longo prazo precisam ser avaliados.

No presente estudo, a maioria dos familiares relatou que as medicações apresentam efeitos colaterais, destacando-se o aumento do apetite e a sedação das crianças, bem como a insônia, a tontura e o ganho de peso. Apesar dos achados, os efeitos colaterais associados à risperidona foram menores ao serem comparados com estudos que fizeram uso do haloperidol (DeFilippis \& Wagner, 2016; Orsolini et al., 2016; Masi, Demayo, Glozier \& Guastella, 2017).

Em relação ao uso do aripiprazol, dados da literatura têm demonstrado eficácia sobre os casos de irritabilidade associado ao TEA, sendo seguro e bem tolerado. Assim podendo ser usado quando comportamentos como acessos de raiva, agressão, comportamento auto lesivo ou uma combinação destes. Porém tem como efeito colateral um ganho de peso. Tais achados estão condizentes com o que foi observado nesse presente estudo, no qual observou-se como efeitos adversos ao uso do aripiprazol comportamentos agressivos e o ganho de peso (Owen et al., 2009; DeFilippis \& Wagner, 2016; Masi, Demayo, Glozier \& Guastella, 2017; Lord, Elsabbagh, Baird \& Veenstra-Vanderweele, 2018).

Quanto aos ISRSs, tais como a clomipramina, fluoxetina, fluvoxamina, sertralina, paroxetina, o citalopram e o escitalopram, destacam-se neste estudo, a fluoxetina e a sertralina. Dados encontrados por Nikolov, Jonker e Scahill (2006), Masi, Demayo, Glozier e Guastella (2017) e Genovese e Butler (2018) apontaram que a fluoxetina se mostrou eficaz em 
comportamento repetitivo e melhora global, enquanto que a fluvoxamina mostrou-se útil no comportamento compulsivo e agressão, bem como no maior comportamento pró-social nos adultos com autismo. No entanto, segundo os dados desses autores, seu uso mostrou resultados negativos com efeitos colaterais de ativação de comportamento (hiperatividade, desinibição, insônia e agressão).

Pesquisas mostraram que o tratamento medicamentoso ainda possui pouca eficácia na redução dos sintomas psiquiátricos associados a condições de desenvolvimento intelectual, principalmente a risperidona e o aripiprazol no tratamento da irritabilidade e o metilfenidato e a atomoxetina na hiperatividade e déficit de atenção (Masi, Demayo, Glozier \& Guastella, 2017; Lord, Elsabbagh, Baird \& Veenstra-Vanderweele, 2018; Cukier \& Barrios, 2019), como foi demonstrado nos resultados do presente estudo.

Práticas de prescrição de psicofármacos são fortemente influenciadas por comorbidades associadas ao TEA, o que pode explicar a grande proporção de psicofármacos prescritos que receberam mais de um psicotrópico. Segundo Murray et al. (2014) os medicamentos mais utilizados para o TEA são drogas psicotrópicas; os medicamentos mais prescritos são medicamentos para dormir (incluindo a melatonina), psicoestimulantes e antipsicóticos. Destaca-se que mais crianças com autismo receberam psicoestimulantes e medicamentos para dormir ao longo do tempo.

Conforme foi demonstrado com os dados desta pesquisa, os medicamentos utilizados no tratamento de crianças com TEA, como a risperidona e o aripiprazol, podem provocar efeitos adversos em uma grande proporção de usuários, como sedação e aumento do apetite. Tais informações estão de acordo com os dados de Leite, Meirelles e Milhomem (2015) e de Masi, Demayo, Glozier e Guastella (2017).

Por conseguinte, observou-se que as mães dos pacientes que possuem ensino superior completo possuíam mais chances de acatar o tratamento não medicamentoso, em comparação às mães que possuíam ensino fundamental completo. Este achado pode ser atribuído ao fato de essas mães terem um maior conhecimento dos benefícios que a terapia não medicamentosa pode proporcionar às crianças com autismo. Entretanto, não foram identificados dados na literatura para comprovar ou refutar essa associação.

A maior parte dos participantes dessa pesquisa relatou que os pacientes utilizavam tratamento não-medicamentoso, sendo esta modalidade considerada o tratamento que proporciona melhor qualidade de vida tanto para as crianças com autismo quanto para os cuidadores. Dados presentes no estudo de Babatin, Alzahrani, Jan, Alkarimi e Jan (2016) mostraram que as crianças com TEA precisam de tratamentos individualizados e intensivos e para isso são necessários centros especializados em autismo que forneçam intervenções comportamentais, psicológicas e educacionais.

Ainda sobre esse aspecto, ressalta-se que no Brasil ainda é escasso no sistema de saúde pública a disponibilidade de pessoas bem treinadas, de financiamento e de infraestrutura para oferecer intervenções analistas comportamentais intensivas, precoces e de alta qualidade aos pacientes com TEA. Além disso, ensinar aos cuidadores as intervenções pode contornar a necessidade de recursos adicionais, como os profissionais treinados e em lugares de difícil acesso aos mesmos. Tais informaç̃os são apresentadas nos estudos de Mello, Ho, Dias e Andrade (2013), Barboza, Silva, Barros e Higbee (2015), Masi, Demayo, Glozier e Guastella (2017), Genovese e Butler (2018) e Barboza, Costa e Barros (2019).

Com essa pesquisa, observou-se que as crianças com autismo necessitam de um apoio para além dos sintomas principais do TEA e aquelas que têm alguma deficiência intelectual (DI) mostraram-se mais propensas a uso de medicamentos e serviços especializados quando comparadas aos que não possuem DI, conforme mostrado nos estudos de Baumer \& Spence (2018), Consolini, Lopes e Lopes (2019), Hodges, Fealko e Soares (2020) e Hyman, Levy e Myers (2020).

Levando em consideração a terapia não medicamentosa avaliada pelos familiares das crianças com TEA, constatou-se que toda criança com autismo precisa de intervenção que forneça de forma sistemática, intensiva e regular uma estrutura organizacional externa que estimule a aplicação das capacidades cognitivas e autorregulatórias da criança em uma ampla 
variedade de tarefas de interação social, pois isso implica em estímulos centrais como enriquecimento perceptivo, comunicativo e linguístico, motor e socioafetivo. Lembrando sempre que a família deve procurar manter sempre um bom contato com os profissionais de saúde, sendo esse um fator de extrema importância e implica a necessidade de um apoio especial para a mesma. Esse fato foi pontuado na pesquisa de Fernandes e Amato (2013) e de Zalaquett, Schönstedt, Angeli, Herrera e Moyano (2015).

Ainda dentro desse âmbito, constatou-se que essa estruturação tem como base a organização do espaço e do contexto da criança e a filtragem das características externas, como traços da personalidade e comportamental, para que a própria criança possa construir uma percepção do mundo e de si com mais coerência, o que tornará possível o desenvolvimento. Esta abordagem baseia-se na teoria da aprendizagem e alicerça-se em estratégias da teoria do comportamento. Assim, o profissional busca, dentro de um quadro terapêutico, a comunicação e a relação com a criança. A observação fina dos comportamentos, a análise da contratransferência, o recurso à atenção flutuante, para usar a noção de Freud, serão alguns instrumentos que permitirão ao terapeuta restituir à criança o que ele compreende dos seus estados psíquicos no decorrer das sessões (Debelle dos Santos, 2005).

De acordo com as informações colhidas nesta investigação, conforme a percepção dos cuidadores, o tratamento medicamentoso pode não promover melhorias no comportamento agressivo das crianças autistas. Tal fato deve-se ao fato de a terapia cognitivo-comportamental (TCC) no TEA apresentar a manutenção de técnicas cognitivas e comportamentais, como psicoeducação, exposição e resposta, reestruturação cognitiva e regulação emocional. Evidências científicas disponíveis na literatura indicaram que a TCC, assim como em diversos transtornos psiquiátricos, apresenta bons resultados em intervenções com os pacientes com TEA e torna-se mais efetiva por introduzir os pais e os cuidadores no processo de cuidar. Usam-se, também, aplicações do treinamento em habilidades sociais no autismo e diferentes tipos de procedimentos vêm sendo adaptados para atingir objetivos específicos da população com TEA (Consolin, Lopes \& Lopes, 2019; Lord, Elsabbagh, Baird \& Veenstra-Vanderweele, 2018).

Por conseguinte, ainda segundo a pesquisa, aqueles pacientes que não fazem uso de medicação apresentam tendência a não perceberem melhorias no comportamento hiperativo e qualidade do sono em comparação aos que fazem. Assim, na visão dos cuidadores, há maior probabilidade de pacientes que aderem ao tratamento não medicamentoso se beneficiarem com a terapia ocupacional - integral sensorial, podendo proporcionar melhoras significativas em seu domínio intelectual.

Assim, segundo Laugeson, Frankel, Mogil e Dillon (2009) os indivíduos com TEA precisam de intervenções adequadas para desenvolverem habilidades sociais em seus ambientes naturais, pois somente a exposição aos modelos sociais adequados não é suficiente, uma vez que os mesmos não costumam aprender com a modelação social e nem com aprendizagem incidental, pois em muitas situações não apresentam atenção para os estímulos sociais. Sendo assim com a inexistência de intervenções adequadas, os autistas apresentam dificuldade para manter contato olho a olho, demonstram menos espontaneidade na comunicação e dificuldade para interagir com os outros.

Em relação a terapia ocupacional integral-sensorial, de acordo com a pesquisa de Sampaio, Loureiro e Gomes (2015) e de Silva, Silva Neto e Freitas (2020) a musicoterapia têm sido outra estratégia em crescimento no tratamento não medicamentoso dos autistas. Estudos realizados na neurociência trouxeram novas luzes sobre a estrutura e funcionamento do sistema nervoso de pessoas com TEA, incluindo o modo como esta população processa a música e devido a essa utilização desses conhecimentos é fornecido novas explicações a respeito do modo pelo qual a música pode ser utilizada no contexto de uma relação terapêutica para promover melhora da saúde bem como para subsidiar novas abordagens clínicas de tratamento, diagnóstico e avaliação do processo terapêutico de pessoas com TEA. Observou-se a eficácia do tratamento musico terapêutico para pessoas com TEA principalmente em relação à interação social e à comunicação. 
Sobre esse aspecto da terapia ocupacional - integral sensorial, segundo dados da pesquisa de Castro e Gonçalves (2013) a atuação de profissionais fonoaudiólogos no desenvolvimento das crianças com TEA é indiscutível, pois os autistas geralmente possuem alterações de linguagem caracterizadas por atrasos significativos ou ausência total de desenvolvimento desta habilidade. A linguagem é tida como a principal forma de interação social, logo a terapia fonoaudióloga precisa ser inserida precocemente para que sejam trabalhados os aspectos mais importantes do autismo, a interação social debilitada. Assim, percebe-se o quão importante é que essa terapia seja incluída precocemente para promover inserção social das crianças com TEA em seu meio social.

Outros pontos positivos da terapia ocupacional - integral sensorial foram documentados na literatura. Nesse aspecto, no estudo de Silva (2019), o mediador escolar entra também como uma estratégia de grande importância no que tange o desenvolvimento dos pacientes com TEA, pois pode contribuir com a redução das barreiras que dificultam a socialização e a permanência da criança autista na escola, em especial dentro da sala de aula regular. Lord, Elsabbagh, Baird e VeenstraVanderweele (2018) também defendem esta ideia.

Outros achados relevantes no estudo de Cruz e Praxedes (2018) incluíram o esporte identificando-se como uma importante estratégia utilizada com possíveis contribuições para o aprimoramento motor de crianças e jovens com Transtorno do Espectro Autista (TEA). A utilização de jogos, práticas esportivas, atividades aquáticas, gincanas, exercícios de fortalecimento muscular, de equilíbrio e psicomotricidade, práticas motoras com trampolins e atividades ao ar livre.

Revisão integrativa realizada por Sousa, Sousa e Bezerra (2021), objetivando identificar as consequências das atividades esportivas para aqueles indivíduos com TEA, constatou muitas benesses decorrentes da prática de atividades esportivas para o grupo, destacando-se implicações positivas de ordem psicossocial $(47,06 \%)$, de natureza física $(47,06 \%)$ e cognitiva $(5,88 \%)$.

Com os dados obtidos com o presente estudo, essas intervenções com a terapia ocupacional - integral sensorial demonstrou diminuição de estereotipias, aumento do repertório motor, desenvolvimento da coordenação motora, do equilíbrio, da agilidade e do controle postural, e melhorias em aspectos sociais e comunicativos. Esses dados são confirmados com os estudos de Genovese e Butler (2018) e de Cruz e Praxedes (2018). Dessa forma, viu-se que a educação física pode contribuir para o desenvolvimento motor de crianças e jovens com TEA por meio de um programa de exercícios especificamente elaborado.

\section{Considerações Finais}

Diante do objetivo desse estudo, ao comparar o tratamento não medicamentoso e medicamentoso nos pacientes com TEA na visão dos cuidadores, observou-se o quão utilizado e benéfico é o tratamento não medicamentoso. Na visão dos cuidadores, pacientes que aderem ao tratamento não medicamentoso se beneficiam com a terapia ocupacional - integral sensorial.

Em contrapartida, pacientes que não fazem uso de medicação apresentam tendência a não perceberem melhorias no comportamento hiperativo e qualidade do sono, em comparação àqueles que fazem. Todavia, o estudo também nos mostrou que o tratamento medicamentoso não tem boas respostas quando relacionado à promoção de melhoria de comportamentos agressivos. Entre as medicações, a que se mostrou mais utilizada foi a risperidona. A maioria dos cuidadores relatou que as medicações apresentam efeitos colaterais, entre estes, o aumento do apetite.

Como foi bem visto no resultado da pesquisa realizada, a associação entre o tratamento medicamentoso e não medicamentoso são capazes de trazer resultados mais positivos para os portadores de TEA. Diante do exposto, fica claro que o tratamento não medicamentoso é de mais valia para o desenvolvimento e para qualidade de vida da criança com TEA, levando a melhorias dos sintomas nucleares, além de que não trazer efeitos colaterais atrelados, como o medicamentoso que pode levar 
a apresentar alguns efeitos adversos em uma grande proporção dos usuários, como sedação e aumento do apetite. Logo, o tratamento medicamentoso é introduzido em busca de melhorias de sintomas específicos que acompanham os sintomas nucleares, ainda que a medicação melhore a vida de alguns pacientes, seus benefícios são restritos.

Haja vista a importância e prevalência do tema, faz-se necessário mais estudos estabelecendo os benefícios entre a associação do tratamento farmacológico para sintomas específicos do autismo com a terapia ocupacional - integral sensorial para uma melhor compreensão de como melhorar a qualidade de vida de crianças com transtorno do espectro autista.

\section{Referências}

American Psychiatric Association. (2013). Diagnostic and Statistical Manual of Mental Disorders, (5a ed.), (DSM-V). American Psychiatric Association.

Babatin, A. M., Alzahrani, B. S., Jan, F. M., Alkarimi, E. H., \& Jan, M. M. (2016). The availability of services for children with autismo spectrum disorder in a Saudi population. Neurosciences (Riyadh, Saudi Arabia), 21(3), 223-226. https://doi.org/10.17712/nsj.2016.3.20150597

Barboza, A. A., Costa, L. C. B., \& Barros, R. S. (2019). Instructional Videomodeling to Teach Mothers of Children with Autism to Implement Discrete Trials: a systematic replication. Temas em Psicologia, 27(3), 795-804, 2019. http://dx.doi.org/10.9788/tp2019.3-14.

Barboza, A. A., Silva, A. J. M. e, Barros, R. da S., \& Higbee T. S. (2015). Effects of instructional videomodeling on the performance of caregivers applying teaching programs to children diagnosed with autism. Acta Comportamentalia: Revista Latína de Análisis del comportamiento, 23(4), 405-421.

Benvenuto, A., Battan, B., Benassi, F., Gialloreti, L. E., \& Curatolo, P. (2016). Effectiveness of community-based treatment on clinical outcome in children with autism spectrum disorders: An Italian prospective study. Developmental neurorehabilitation, 19(1), 1-9. https://doi.org/10.3109/17518423.2013.864716.

Brentani, H., Paula, C. S., Bordini, D., Rolim, D., Sato, F., Portolese, J., Pacifico, M. C., \& McCracken, J. T. (2013). Autism spectrum disorders: an overview on diagnosis and treatment. Revista brasileira de psiquiatria, 35(1), S62-S72. https://doi.org/10.1590/1516-4446-2013-S104

Bosa, C A. (2006). Autismo: intervenções psicoeducacionais. Revista Brasileira de Psiquiatria, 28(1), s47-s53.

Castro, M. S. J., Gonçalves, C. A. B. (2013). Proposals for speech therapy for autist children: a systematic review of literature. Distúrbios da Comunicação, 25(1), 15-25.

Consolini, M., Lopes, E. J., \& Lopes, R. F. F. (2019). Terapia Cognitivo-comportamental no Espectro Autista de Alto Funcionamento: revisão integrativa. Revista Brasileira de Terapias Cognitivas, 15(1), 38-50. https://dx.doi.org/10.5935/1808-5687.20190007

Cukier, S., \& Barrios, N. (2019). Intervenciones farmacológicas en discapacidad intelectual y autismo [Pharmacological interventions for intellectual disability and autism]. Vertex (Buenos Aires, Argentina), XXX(143), 52-63.

Cruz, M. R. da; Praxedes, J. (2018). A importância da educação física para o desenvolvimento motor de crianças e jovens com transtornos do espectro autista. e-Mosaicos, 7(14), 187-199.

Debelle dos Santos, F. (2005). Autismo e psicologia clínica de abordagem dinâmica numa sala TEACCH: reflexões e partilha duma prática. Revista Portuguesa de Psicossomática. 7(1-2), 207-217.

DeFilippis, M., \& Wagner, K. D. (2016). Treatment of Autism Spectrum Disorder in Children and Adolescents. Psychopharmacology bulletin, 46(2), 18-41.

Elsabbagh, M., Divan, G., Koh, Y., Kim, Y. S., Kauchali, S., Marcín, C., Montiel-Nava, C., Patel, V., Paula, C. S., \& Wang, C. (2012). Global Prevalence of Autism and Other Pervasive Developmental Disorders. Autism Research, 5(3), 160-179, 2012. http://dx.doi.org/10.1002/aur.239.

Fernandes, F. D. M., \& Amato, C. A. de la H. (2013). Applied Behavior Analysis and Autism Spectrum Disorders: literature review. Communication Disorders, Audiology and Swallowing (CoDAS). 25(3), 289-296.

Fombonne E. (2009). Epidemiology of pervasive developmental disorders. Pediatric research, 65(6), 591-598. https://doi.org/10.1203/PDR.0b013e3181 $9 \mathrm{e} 7203$

Genovese, A., \& Butler, M. G. (2020). Clinical Assessment, Genetics, and Treatment Approaches in Autism Spectrum Disorder (ASD). International Journal Of Molecular Sciences, 21(13), 1-18. http://dx.doi.org/10.3390/ijms21134726.

Gerhardt, T. E., \& Silveira, D. T. (2009). Métodos de pesquisa Editora da UFRGS.

Hyman, S. L., Levy, S. E., \& Myers, S. M. (2019). Identification, Evaluation, and Management of Children With Autism Spectrum Disorder. Pediatrics, 145(1), 1-71. http://dx.doi.org/10.1542/peds.2019-3447.

Laugeson, E. A., Frankel, F., Mogil, C., \& Dillon, A. R. (2009). Parent-assisted social skills training to improve friendships in teens with autism spectrum disorders. Journal of autism and developmental disorders, 39(4), 596-606. https://doi.org/10.1007/s10803-008-0664-5

Leite, R., Meirelles, L. M. A., Milhomem, D. B. (2015). Drugs used in the psychotherapeutic treatment of autistic children in Teresina-PI. Informative Geum Bulletin, 6(3), 91-97.

Lord, C., Elsabbagh, M., Baird, G., Veenstra-Vanderweele, J. (2018). Autism spectrum disorder. The Lancet, 392(10146), 508-520. http://dx.doi.org/10.1016/s0140-6736(18)31129-2 
Magalhães, J. M., Silva, T. M. da, Silva, F. da C., Alencar, M. de F. B. de, Rêgo Neta, M. M., Alencar, D. de C., \& Arisawa, E. A. L. S. (2021). Profile of children with autism spectrum disorder. Research, Society and Development, 10(4), e3710413880. https://doi.org/10.33448/rsd-v10i4.13880

Masi, A., Demayo, M. M., Glozier, N., Guastella, A. J. (2017). An Overview of Autism Spectrum Disorder, Heterogeneity and Treatment Options. Neuroscience Bulletin, 33(2), 183-193, 2017. http://dx.doi.org/10.1007/s12264-017-0100-y

Mello, A. M., Ho, H., Dias, I., \& Andrade, M. (2013). Retratos do autismo no Brasil, AMA.

Muhle, R., Trentacoste, S. V., \& Rapin, I. (2004). The genetics of autism. Pediatrics, 113(5), e472-e486. https://doi.org/10.1542/peds.113.5.e472

Murray, M. L., Hsia, Y., Glaser, K., Simonoff, E., Murphy, D. G., Asherson, P. J., Eklund, H., \& Wong, I. C. (2014). Pharmacological treatments prescribed to people with autism spectrum disorder (ASD) in primary health care. Psychopharmacology, 231(6), 1011-1021. https://doi.org/10.1007/s00213-013-3140-7

Newsom, C.; Hovanitiz, C. A. (2006). Autistic spectrum disorders. In E. J. Mash, \& R. A. Barkley (Eds.), Treatment of childhood disorders. New York: Guilford Press, 3, 455-511.

Nikolov, R., Jonker, J., \& Scahill, L. (2006). Autismo: tratamentos psicofarmacológicos e áreas de interesse para desenvolvimentos futuros. Revista Brasileira de Psiquiatria, 28(1), s39-s46.

Orsolini, L., Tomasetti, C., Valchera, A., Vecchiotti, R., Matarazzo, I., Vellante, F., Iasevoli, F., Buonaguro, E. F., Fornaro, M., Fiengo, A. L., Martinotti, G., Mazza, M., Perna, G., Carano, A., De Bartolomeis, A., Di Giannantonio, M., \& De Berardis, D. (2016). An update of safety of clinically used atypical antipsychotics. Expert opinion on drug safety, 15(10), 1329-1347. https://doi.org/10.1080/14740338.2016.1201475

Owen-Deschryver, J. S., Carr, E. G., Cale, S. I., Blakeley-Smith, A. (2008). Promoting Social Interactions Between Students With Autism Spectrum Disorders and Their Peers in Inclusive School Settings. Focus On Autism And Other Developmental Disabilities, 23(1), 15-28. http://dx.doi.org/10.1177/1088357608314370.

Owen, R., Sikich, L., Marcus, R. N., Corey-Lisle, P., Manos, G., McQuade, R. D., Carson, W. H., \& Findling, R. L. (2009). Aripiprazole in the treatment of irritability in children and adolescents with autistic disorder. Pediatrics, 124(6), 1533-1540. https://doi.org/10.1542/peds.2008-3782

Postorino, V., Kerns, C. M., Vivanti, G., Bradshaw, J., Siracusano, M., \& Mazzone, L. (2017). Anxiety Disorders and Obsessive-Compulsive Disorder in Individuals with Autism Spectrum Disorder. Current Psychiatry Reports, 19(12), 1-14. http://dx.doi.org/10.1007/s11920-017-0846-y.

Sampaio, R. T., Loureiro, C. M. V., Gomes, C. M. A. (2015). A Musicoterapia e o Transtorno do Espectro do Autismo: uma abordagem informada pelas neurociências para a prática clínica. Per musi, 32, 137-170.

Silva, L. F. da, Silva Neto, F. S. da, \& Freitas, G. D. de M. (2020). The therapeutic effects of musicalization in children with Autistic Spectrum Disorder (TEA): A literature review. Research, Society and Development, 9(8), e299985399. https://doi.org/10.33448/rsd-v9i8.5399.

Silva, S. R. de A. M. (2019). Mediação escolar no transtorno de espectro autista: abordagem na sala de recursos multifuncional. Revista Educação Pública, 19(6).

Schwartzman, J. S., \& Araújo, C. A. de. (2014). Transtornos do espectro do autismo. Editora Memnon.

Sousa, M. N. A., Sousa, M. N. A., \& Bezerra, A. L. D. (2021). Atividades esportivas para indivíduos com transtorno do espectro autista. Revista Brasileira de Educação em Saúde, 11(1), 90-96.

Zalaquett, D., Schönstedt, M., Angeli, M., Herrrera, C., \& Moyano, A. (2015). Fundamentos de la intervención temprana en niños con trastornos del espectro autista. Revista Chilena de Pediatría, 86(2), 126-131. 2015 http://dx.doi.org/10.1016/j.rchipe.2015.04.025. 